\title{
Epidemiological study of Aspergillosis in chickens and human contacts in chicken farms at Kalyoubia Governorate
}

\author{
Lobna, M.A. Salem and Abdel Fatah,Ali \\ Dept. of Zoonoses. Fac. Vet. Med, Benha University \\ Dept. of Clinical Pathology, Fac. Vet. Med, Benha University
}

\begin{abstract}
A total of 100 suspected chicken lung tissues and 80 environmental samples including litter, feed, water and air (20 samples of each) were collected from different private chicken farms at kalyoubia Governorate, Moreover 30 sputum and 30 blood serum samples were collected from occupationally human contacts with these farms and with chronic respiratory disease. All samples except serum were examined mycologically but serum samples were serologically examined by ELISA. The percentage of Aspergillus species was $24 \%, 55 \%, 55 \%, 10 \%, 50 \%$ and $23.3 \%$ in lung tissues, feed, litter, water, air and human sputum respectively. Almost all Aspergillus species isolated from lung tissues of chickens were also recovered from environmental samples and human sputum samples but Aspergillus fumigatus was the predominant and the most pathogenic fungus isolated from all samples followed by Aspergillus niger and Aspergillus flavus. The occurrence of infection was high in hot and humid seasons and was considerably high in chickens reared on saw dust litter as compared to rice husk litter. Regarding to human beings, Aspergilli were isolated from all age groups but the majority of infection exists among the 20-40 years group (26.7\%) but it was nearly equal in both males (23.5\%) and females (23.1\%) and higher in farm workers (29.4\%) than in farm owners and veterinarians. Aspergillus fumigatus was detected in sputum samples with $13.3 \%$ but in blood serum samples by using ELISA $\operatorname{IgG}$, it was 10\%. The zoonotic importance of Aspergillosis as occupationally disease among poultry farm workers as well as the suggested hygienic measures were discussed to decrease its transmission to humans.
\end{abstract}

Keywords: Epidemiology, Aspergillosis, Chicken farms , Human Contacts ,Egypt

\section{Introduction}

In recent years, Fungal infections have emerged as a world-wide health care and has become an important cause of respiratory infection in humans owing to extensive use of broad spectrum antibiotics, corticosteroids and immune-suppressive agents and increasing population of terminally ill and debilitated patients(1) which trigger an interest to examine the source and reservoir of such fungi. Aspergillus infection is the commonest invasive fungal infection which involves respiratory tract. It can be ascribed to the presence of the fungal spores in soil, water, decaying vegetation and indoor environment (2). Aspergillosis has emerged as a significant poultry health concern for poultry producers and humans health officials as it causes economic losses in poultry industry in Egypt, moreover, Aspergillus species have been isolated from many pulmonary infections in human. Aspergillosis is the most important fungal infection being caused by Aspergillus fumigatus in particular, is the most important air borne saprophytic fungus and rarely by Aspergillus flavus or Aspergillus niger. The disease mainly affects respiratory tract of birds and develops as broncho pneumonia. The clinical signs such as dyspnea, gasping, cyanosis and hyperemia are usually associated with the disease. However, the affected birds normally don't produce respiratory noise associated with other respiratory problems (3). Moreover,Aspergillosis primarily causes high morbidity and mortality especially in young chicks (4) . Aspergilli can be isolated from environmental samples and are world wide in distribution due to the spores of this fungal pathogens are resistant in nature and the birds as well as people who work with them, coming in contact with spores through contaminated feed or litter and may get affected after inhaling the spores (5). Poultry farm workers are at high risk of developing respiratory problems as they are exposed to high levels of dust containing Aspergillus spores during handling and processing of contaminated materials ( 6). Depending on the host immunity, lung structure and degree of inoculums, the Patients may present with some non specific symptoms as fever ,anorexia, malaise ,cough and dyspnea. In some cases hemoptysis might occur following vascular invasion by Aspergillus filaments. Meanwhile, other cases may present by pulmonary infarction with chest pain and heaviness (7). On the other hand, several species of Aspergillus produce toxins which have various effects such as Aflatoxins that are carcinogenic and cause hepatic and kidney damage or chronic damage of human bones (8). The diagnosis of Aspergillus infection presents considerable difficulty as the signs and symptoms in most cases of Aspergillosis are non-specific, and radiological findings are of little diagnostic help, So the diagnosis of Aspergillosis requires the isolation and identification of the fungus. Serologic assays are now being developed as an attempt to allow for the rapid and specific diagnosis of Aspergillus infections. So the aim of this study was to determine the epidemiology of Aspergillus species in some chicken farms and to assess the 
occupational exposure of chicken farm workers to Aspergillus with possible adverse health effects with special reference to the available preventing measures to overcome such problem.

\section{1. Collection of samples}

\section{Materials and Methods}

The present study was carried out to ascertain the incidence of Aspergillosis in private chicken farms at kalyoubia governorate during different seasons. On the basis of clinical signs and history narrated by poultry farmers, the affected dead birds were subjected to post mortem examination and a total of 100 lung tissue samples were collected from suspected lungs showing lesions (necrosis and granulomatous swelling).In addition to 80 environmental samples including litter samples, feed samples, air samples and water samples ( 20 per each) were collected. Moreover, 30 sputum samples and 30 blood serum samples were collected from the occupationally human contacts with these farms and with the history of cough, chest pain and chronic respiratory disease. All samples were aseptically transported to the laboratory of zoonoses department, Benha University and were prepared according to (9).

\section{2. Mycological examination for Aspergillus species}

All samples except blood serum were directly streaked on Sabouraud's dextrose agar containing chloramphenicol (50 mg per liter) to control bacterial contamination for isolation of fungus. The suspected Aspergillus colonies were cultured on Czapeck- Dox agar for final macroscopic and microscopic identification according to (10\&11).

\section{3. Serological examination of human serum samples}

Enzyme Linked Immunosorbant Assay ( ELISA) for detection of IgG qualitative and quantitative Aspergillus fumigatus (IBL-RE56111) Hamburg, Germany) has been done on serum samples of human according to instructions at Molecular Biology Unit, Faculty of Medicine, Benha University.

\section{Results and Discussion}

Table. 1: Detection of Aspergillus species in lungs and environmental samples

\begin{tabular}{|c|c|c|c|c|c|c|c|c|c|}
\hline \multirow[t]{3}{*}{ Samples } & \multirow{3}{*}{$\begin{array}{c}\text { Examined } \\
\text { number }\end{array}$} & \multicolumn{6}{|c|}{ Positive isolates } & \multicolumn{2}{|c|}{ Total } \\
\hline & & \multicolumn{2}{|c|}{ A. fumigatus } & \multicolumn{2}{|c|}{ A. niger } & \multicolumn{2}{|c|}{ A. flavus } & & \\
\hline & & No & $\%$ & No & $\%$ & No & $\%$ & No & $\%$ \\
\hline Lungs & 100 & 13 & 13 & 8 & 8 & 3 & 3 & 24 & 24 \\
\hline Feed & 20 & 4 & 20 & 3 & 15 & 4 & 20 & 11 & 55 \\
\hline Litter & 20 & 6 & 30 & 3 & 15 & 2 & 10 & 11 & 55 \\
\hline Water & 20 & 1 & 5 & $\mathbf{0}$ & $\mathbf{0}$ & 1 & 5 & 2 & 10 \\
\hline Air & 20 & 5 & 25 & 3 & 15 & 2 & 10 & 10 & 50 \\
\hline
\end{tabular}

Table. 2: Occurrence of Aspergillus species in different types of litter

\begin{tabular}{|c|c|c|c|c|c|c|}
\hline \multirow[t]{2}{*}{ Type of litter } & \multirow[t]{2}{*}{ Examined No } & \multicolumn{2}{|c|}{ Positive isolates } & \multirow[b]{2}{*}{ A. flavus } & \multicolumn{2}{|c|}{ Total } \\
\hline & & A. fumigatus & A.niger & & $+v e$ & $\%$ \\
\hline Saw dust & 10 & 4 & 2 & 1 & 7 & 70 \\
\hline Rice husk & 10 & 2 & 1 & 1 & 4 & 40 \\
\hline Total & 20 & 6 & 3 & 2 & 11 & 55 \\
\hline
\end{tabular}

Table 3: Distribution of positive Aspergillus species isolates in different seasons

\begin{tabular}{lcccc}
\hline Seasons & \multicolumn{2}{c}{ Lung isolates (24) } & \multicolumn{2}{c}{ Environmental isolates (34) } \\
& + ve & $\%$ & + Ve & $\%$ \\
Winter & 7 & 29.2 & 10 & 29.4 \\
Spring & 2 & 8.3 & 3 & 8.8 \\
Summer & 12 & 50 & 14 & 41.2 \\
Autumn & 3 & 12.5 & 7 & 20.6 \\
\hline
\end{tabular}

Table. 4: Detection of Aspergillus in human samples by culturing and serology

\begin{tabular}{lcccc}
\hline Isolates & Culturing of sputum & $(30)$ & ELISA IgG (30) & \\
& + ve & $\%$ & + Ve & 10 \\
A.fumigatus & 4 & 13.3 & 3 & \\
A.niger & 2 & 6.7 & & \\
A.flavus & 1 & 3.3 & & \\
Total & 7 & 23.3 & & \\
\hline
\end{tabular}


Epidemiological study of Aspergillosis in chickens and human contacts in chicken farms at .....

Table. 5: Distribution of positive Aspergillus species isolates in human sputum samples

\begin{tabular}{|c|c|c|c|c|c|}
\hline \multirow[b]{2}{*}{ Variable } & \multicolumn{5}{|c|}{ Positive Isolates } \\
\hline & $\begin{array}{c}\text { Examined No } \\
(30)\end{array}$ & $\begin{array}{c}\text { A. fumigatus } \\
\text { (4) }\end{array}$ & $\begin{array}{c}\text { A.niger } \\
\text { (2) }\end{array}$ & $\begin{array}{l}\text { A. flavus } \\
\text { (1) }\end{array}$ & total \\
\hline \multicolumn{6}{|l|}{ Age } \\
\hline $15-20$ years & 6 & 1 & $\mathbf{0}$ & $\mathbf{0}$ & $1(16.7 \%)$ \\
\hline $20-40$ years & 15 & 2 & 1 & 1 & $4(26.7 \%)$ \\
\hline $\begin{array}{c}>40 \text { years } \\
\text { sex }\end{array}$ & 9 & 1 & 1 & $\mathbf{0}$ & $2(22.2 \%)$ \\
\hline Male & 17 & 2 & 1 & 1 & $4(23.5 \%)$ \\
\hline Female & 13 & 2 & 1 & $\mathbf{0}$ & $3(23.1 \%)$ \\
\hline \multicolumn{6}{|l|}{ Occupations } \\
\hline Farm workers & 17 & 3 & 1 & 1 & $5(29.4 \%)$ \\
\hline Farm owners & 8 & 1 & 1 & $\mathbf{0}$ & $2(25 \%)$ \\
\hline Veterinarians & 5 & $\mathbf{0}$ & $\mathbf{0}$ & $\mathbf{0}$ & $0(0 \%)$ \\
\hline
\end{tabular}

The results recorded in table (1) illustrated that out of 100 examined suspected lung tissues of chickens, the percentage of Aspergillus species was $24 \%$. Moreover, the samples collected from the environment (feed, litter, water \& air) were found to be polluted with Aspergillus species with a percentage of $55 \%, 55 \%, 10 \% \& 50 \%$ respectively. These results substantiate what had been reported by $(12,13 \& 14)$, but lower than recorded by (15\&16). The difference can be accepted due to several factors as the density of birds, topographic conditions and other ambient conditions (from hot humid to cold dry). These findings denotes the role of chickens in Aspergillus epidemiology and may play a dangerous role in prevalence of Aspergilli in slaughtered and frozen chickens (17). Moreover, Aspergilli were isolated from environmental samples as spores of this fungal pathogen are resistant in nature and indicates that the litter and feed may probably play an important role in dissemination of the disease. It is of interest that (6) mentioned that Aspergillus infection is acquired by exposure of susceptible human or avian hosts to the contaminated environment and inhalation of Aspergillus spores. In addition to, the predisposing factors for flaring spore generation and dissemination in the environment or air include warm environment, humidity, poor ventilation and sanitation along with storage of feed (18). From the previously obtained results, it is clear that the incidence of A.fumigatus isolation was (13\%) which is more than A.niger (8\%) and A.flavus (3\%) in chicken lungs. Also from environmental samples, A. fumigatus isolates were higher than other Aspergillus species.This may be attributed to the spores of A.fumigatus are smaller than those of other Aspergillus species (19) and they added that, A. fumigatus can grow in litter of poor quality, in feed stored in poor conditions and in air from infected broiler farms. Furthermore, inadequate ventilation increases the risk of exposure of susceptible host to inhalation of spores causing high morbidity and mortality.

Regarding to the occurrence of Aspergillus in different types of litter. Table (2) showed that the incidence of Aspergillosis was considerably higher in farms with saw dust as litter (70\%) as compared with farms with rice husk litter (40\%). This is in agreement with (15) who detected Aspergillosis with $68.7 \%$ and $32.2 \%$ in both types of litter respectively. It is speculated that the saw dust is more favorable for the fungal growth as compared to rice husk because of its higher moisture contents, allowing the fungal growth.

Concerning the distribution of Aspergillosis among different seasons, table (3) indicated that the disease is prevailing throughout the year but in hot and humid weather condition, the percentage of positive isolates in both lung and environmental samples was higher in summer $(50 \% \& 41.2 \%)$ respectively, followed by winter season which it was $(29.2 \% \& 29.4 \%)$ respectively as compared with other seasons of the year. These results are in accordance with (20\&15)who reported that Aspergillosis is a threat of poultry health because of the warm, humid environment and the disease in winter season is common when indoor gas levels tend to be highest.

In general, poultry is constantly exposed to these fungi in its environment and the predisposing factors for flaring spore generation and dissemination in the air and environment include long exposition and highly contaminated environment and litter, high humidity in poultry houses, poor ventilation, malnutrition and stress (18\&20).

The results presented in table (4) showed that Aspergillus species were isolated from 7(23.3\%) out of 30 sputum samples collected from persons in close contact with chicken farms with chronic pulmonary diseases. This substantiated what has been reported by (12) $27.5 \%$, (6) $22 \%$ and (22) $29 \%$ but it is higher than that obtained by (23) $4.8 \%$ and lower than recorded by (24) $45 \%$. This discrepancy may be due to massive population growth and urban development which increased the prevalence of fungal infections in certain areas and putting more people at risk (25). Susceptibility to Aspergillus appears to be enhanced by certain factors like therapy with antibiotics and chemotherapeutic drugs and steroids, as antibiotics and steroids may stimulate the growth and virulence of infecting fungus by destruction of competing bacterial flora. Common use of broad spectrum antibiotics in the pulmonary disease for long duration, for example in pulmonary tuberculosis, lung 
abscess, and chronic bronchitis, depression of bone marrow activity, carcinoma and leukaemia may predispose to fungal infection .

Consulting the previously obtained results (table 1\&4), it is clear that the incidence of A. fumigatus isolation from lung tissues of chickens, environmental and sputum samples surpass A. niger and A. flavus ,this denoted that there is a possibility to induce infection by the three Aspergilli but A.fumigatus was the most pathogenic for chickens and humans .

Table (4) revealed that the incidence of Aspergillus fumigatus in humans by culturing of sputum and serology by using ELISA IgG was $13.3 \%$ and $10 \%$ respectively. The isolation of Aspergillus from sputum is often considered a contaminant. However, seropositivity detected in the present study correlates with results of similar studies by (26\&27) and suggests that serological tests have an advantage over routine culture for diagnosis of pulmonary Aspergillosis. Detection of antibodies indicates that there is definite infection induced antibody production and thus rules out the presence of fungus as mere contaminant. Pulmonary Aspergillosis occurs in immunocompromised hosts in whom immune response is less or may be absent (28) and in such cases serological findings may be absent. Therefore, such cases with negative serological findings may not be considered negative for infection. Thus a combination of culture and serology can be ideal to confirm the diagnosis of pulmonary Aspergillosis.

From results in table (5), it is evident that the majority of Aspergilli infection exists among the 20-40 years group ( $26.6 \%$ ) more than among $15-20$ years group (16.7\%) and among more than 40 years group $(22.2 \%)$. It appears to be associated with the actual work age of Egyptian workers at these farms. Concerning the sex distribution, the occurrence of Aspergilli was nearly equal in both males ( $23.5 \%)$ and females $(23.1 \%)$. This may attributed to all family were working and living near those farms, so the risk of infection is equal. Regarding to occupation, the distribution of Aspergillosis was higher in farm workers (29.4\%) followed by farm owners $(25 \%)$ but negative in veterinarians $(0 \%)$. This may be due to actual work conditions as preparing feed, cleaning farms, performing veterinary treatment and the lower level of personal hygiene and standard of body health which are predisposing factors in human Aspergillosis.

\section{Conclusion}

From this study, it can be concluded that the human beings and chickens may share the hazard of contracting Aspergillosis and the presence and distribution of Aspergillus species in poultry farms suggests a possible occupational health problem and the predisposing factors play an important role in such affection. Due to the ubiquitous nature of the fungus, it is impossible to establish practical control measures. It is advisable to take special precautionary measures as destruction of affected chickens hygienically, avoidance of damp litter and dust by efficient ventilation, mouldy bedding or feed shouldn't be handled or given to birds and hygienic conditions in incubators and incubation rooms are essential to decrease the hazard of spore inhalation which causing infection in both chickens and humans, so increase the awareness for preventive and protective measures is essential to avoid exposure of occupationally workers to infection.

\section{Reference}

[1]. D. Biswas, S. Agarwal, G. Sindhwani,and J. Rawat,Fungal colonization in patients with chronic respiratory diseases from Himalayan region of India, Annals of Clinical Microbiology and Antimicrobials, 9(28), 2010,1-7.

[2]. Z. Xiao-dong, H. Xiao-peng, Y. Hang, W. Wei, Z.Xin, M. Lin-lin, et al, Aspergillus pneumonia in renal transplant recipients, Chinese Medical Journal ,121(9), 2008,791-94.

[3]. C. Milos,V.Kapetanov, andV.Dubravka ,Investigation of dissemination of Aspergillosis in poultry and possible control measures Proc.Nat. Sci, Matica Srpska Novi Sad (120),2011,267-276.

[4]. K.Dhama, S.Chakraborty, A.K.Verma, R.Tiwari, R. Barathidasan, A.Kumar, and S.D. Singh,Fungal mycotic diseases of poultry. Diagnosis,Treatment and Control ,Areview.Pakistan Journal of Biological Sciences, 2013, 1-15.

[5]. K.R.Dhama, R.Barathidasan,R.Tiwari,and S.D. Singh, Aspergillosis, An important fungal disease of poultry and other birds, Poult, World,( 9),2012,7-9.

[6]. R.Sabino, V.M Faisca, E. Carolino,C.Verissimo,and C.Viegas, Occupational exposure to Aspergillus by swine and poultry farm workers in Portugal, Journal of Toxicology and environmental health . part A,(75), 2012,1381-1391.

[7]. L.Fouad, M.S.Fathi, and A. ElMaragy, The clinical relevance of aspergillus isolation from respiratory tract samples and detection of aspergillus galactomannan antigen in serum of patients with acute exacerbation of COPD, Natural and Science,11(1),2013, 40-43.

[8]. P. N Acha, and B.Szyfres, Zoonoses and communicable diseases common to man and animals. "Aspergillosis" 3 rd Ed.( 1), 2006,305-310.

[9]. M.R. Mcginnis, Laboratory hand book of medical mycology, J. Med. Microbiol, 1(5 ), 1990, 399401

[10]. D.A. Stevens, Diagnosis of fungal infections, Current status, J. Anti microb. Chemother, (49),2002, 11-19.

[11]. N.McClenny,Laboratory detection and identification of Aspergillus species by microscopic observation and culture, The traditional approach,Medical Mycology Supplemen 1,(43),2005,125-128.

[12]. A.A. El- Bassiouni, A.S.El- Ged, A. E.dris, and A.M.Khalid, Aspergillus species isolated from chickens and related human workers, Alex, J. vet. Sci, 4 (2) 1988, 205-211.

[13]. M.K.Refai, S.A. Attia, R.M. Salem, and E.M. Al- Dahshan, Studies on the pathogenicity and enzymatic activities of Aspergillus fumigatus, A.Flavus and A. niger isolated from chickens and their environment, Egypt. J. comp. Path \& clinic. Path. 17( 2), 2004,193- 205.

[14]. G .Andreia Spanamberg, G.Renata; S.Gabriela Miller and F. Cibele Floriano, Aspergillus fumigatus from normal and condemned carcasses with air Sacculitis in commercial poultry, Pesqu. Vet. Bras. 33(9), 2013,1071-1075. 
[15]. M.A.Sajid, A. Khan, and V.Rauf, Aspergillus fumigatus in commercial poultry flocks, a serious threat to poultry industry in Pakistan, J. Anim. PI. Sci. 16(3-4), 2006, 79-81.

[16]. D.Lorin, Evaluation of fungal incidence in broiler farm, Scientific works series C. veterinary medicine, vol. LIX (1) ,2013, 105-109.

[17]. A.M. Edris, Mycological evaluation of frozen meat and chicken, ph. D., Fac. Vet. Med, 1986 Alexandria University .

[18]. A.R.Khosravi, T. Shokri, A.R.Ziglari, Z. Naeini, Mousavi and H.Hashemi,Out break of severe disseminated Aspergillosis in aflock of ostrich, Mycoses(51),2008, 557-559.

[19]. P. Arne, and S.Thierry, Aspergillus fumigatus in poultry,International Journal of Microbiology Article, ID746356, 2011,14 pages.

[20]. J. L., Richard, Aspergillosis in disease of poultry 9 th ed. Iowa state university press. Ames, Iowa, 1991, 326-334.

[21]. R.Whyte, Occupational exposure of poultry stockmen in current barn systems for egg production in the United Kingdom. Br. Poult. Sci. (43),2010,364-373.

[22]. G.P Shrimali, J.K.Bhatt, R. Rajat, R.V. Parmar S. Nayak, and D.Chandralekha, Isolation of Aspergillus species from human samples, Astudy conducted in a tertiary care hospital, National Journal of medical research,3 (3) ,2013, 289-291.

[23]. K.J.Pursell, and J. Paredes, The incidence of colonization and invasive disease in a population of AIDs patients. International conference AIDs June 22 (1), 1990, 237.

[24]. T.Tashiro, K. Izumokawa, M.Tashiro, T.Takazono, Y. Morinage, K.Yamamoto , Y.Imamura, and T.Miyazaki, Diagnostic significance of Aspergillus species isolated from respiratory samples in an adult pneumologyward, Med, Mycol, (49), 2011,581587.

[25]. D.E. Schraufnagel, Fungal lung diseases, Chapter 9, 2009, 89-97.

[26]. P. Vijayachari, N. Lukshmi, and A.G. Kumar, Serodiagnosis of pulmonary Aspergillosis, Indian J Med Microbiology ,15(1), 1997,23-24.

[27]. A.M. Kurhade, J. M. Deshmukh, R. P. Fule, C.Chande, and S. Akulwar, Mycological and serological study of pulmonary Aspergillosis in central India, Indian Journal of medical microbiology, 2 (3), 2002, 141- 142.

[28]. R.C. Young,and J.E Bennett, Invasive aspergillosis:absence of detectable antibody response. Am J, respire, Dis , (104), 1971,710716. 